\title{
Multiple furunculoid myiasis in an infant
}

\section{Khadim Diop', Aminata Mbaye ${ }^{2}$, Mame Téné Ndiaye Diop', Fatou Diasse', Saer Diadie², Aminata Deh ${ }^{3}$, Niare Ndour ${ }^{3}$, Coumba Ndiaye ${ }^{3}$, Mamadou Sarr ${ }^{3}$, Boubacar Ahy Diatta ${ }^{3}$, Maodo Ndiaye ${ }^{3}$, Moussa Diallo ${ }^{3}$, Suzanne Oumou Niang ${ }^{3}$}

${ }^{\text {}}$ Service De Dermatologie, Hôpital D'enfants Albert Royer, Dakar Sénégal, ${ }^{2}$ Service De Pédiatrie, Hôpital D'enfants Albert Royer, Dakar, Sénégal, ${ }^{3}$ Service De Dermatologie, Hôpital Aristide Le Dantec, Dakar, Sénégal

Corresponding author: Khadim Diop, MD, E-mail: bambadiop100391@gmail.com

\begin{abstract}
Subcutaneous or furunculoid myiasis is an ectoparasitosis caused by the cutaneous or subcutaneous carriage of larvae of various diptera. The causal agent is most often Cordylobia Anthropophaga in tropical areas. We report an observation of multiple subcutaneous myiasis in an infant. The toddler was 2 months old, with no previous history of myiasis and was up to date with vaccinations. He was treated for furunculoid lesions evolving for 5 days. The dermatological examination revealed three inflammatory nodules centred by a whitish orifice without fluid discharge, located on the thorax, the back and the buttocks. The rest of the clinical examination was unremarkable. A maggot was extracted by bi-digital compression of a nodule in the thorax. The diagnosis of furunculoid myiasis was set. The treatment consisted of manual extraction of the maggots and local care. The evolution was favourable after two weeks, labelled by a complete healing of the lesions. Our case is particular because of the occurrence of subcutaneous myiasis in an infant and its misleading clinical presentation simulating multiple furuncles.
\end{abstract}

Key words: Subcutaneous myiasis; Infant; Furuncles.

How to cite this article: Diop K, Mbaye A, Diop MTD, Diasse F, Diadie S, Deh A, Ndour N, Ndiaye C, Sarr M, Diatta BA, Ndiaye M, Diallo M, Niang SO. Multiple furunculoid myiasis in an infant. Our Dermatol Online. Our Dermatol Online. 2022;13(Supp. 1):15-19.

Submission: 20.06.2021; Acceptance: 01.10.2021

DOI: 10.7241 /ourd.2022S1.3 


\title{
Des myiases furonculoïdes multiples chez un nourrisson
}

\section{Khadim Diop', Aminata Mbaye ${ }^{2}$, Mame Téné Ndiaye Diop', Fatou Diasse', Saer Diadie², Aminata Deh ${ }^{3}$, Niare Ndour ${ }^{3}$, Coumba Ndiaye ${ }^{3}$, Mamadou Sarr ${ }^{3}$, Boubacar Ahy Diatta ${ }^{3}$, Maodo Ndiaye ${ }^{3}$, Moussa Diallo ${ }^{3}$, Suzanne Oumou Niang ${ }^{3}$}

\author{
${ }^{1}$ Service De Dermatologie, Hôpital D'enfants Albert Royer, Dakar Sénégal, ${ }^{2}$ Service De Pédiatrie, Hôpital D'enfants Albert \\ Royer, Dakar, Sénégal, ${ }^{3}$ Service De Dermatologie, Hôpital Aristide Le Dantec, Dakar, Sénégal \\ Corresponding author: Khadim Diop, MD, E-mail: bambadiop100391@gmail.com
}

\begin{abstract}
RÉSUMÉ
La myiase sous cutanée ou furonculoïde est une ectoparasitose causée par le cheminement cutané ou sous cutané de larves de divers diptères. Lagent causal est le plus souvent Cordylobia anthropophaga en zone tropical. Nous rapportons une observation de myiases sous cutanée multiples chez un nourrisson. Il s'agissait d'un nourrisson âgé de 2 mois reçu pour des lésions furonculoïdes évoluant depuis 5 jours. Lexamen dermatologique retrouvait trois nodules inflammatoires centrés par un orifice blanchâtre ne laissant pas sourdre de liquide, localisés au niveau du thorax, le dos et la fesse. Le reste de l'examen clinique était normal. La compression bi-digitale d'un nodule au niveau du thorax avait permis l'extraction d'un asticot. Le diagnostic de myiase furonculoïde était retenu. Le traitement consistait à l'extraction manuelle des larves et des soins locaux. Lévolution était favorable au bout de deux semaines, marquée par une cicatrisation complète des lésions. Notre observation est particulière par la survenue de myiases sous cutané chez un nourrisson et sa présentation clinique trompeuse simulant des furoncles multiples.
\end{abstract}

Mot sclés: Myiase sous cutané; Nourrisson; Furoncles

\section{INTRODUCTION}

La myiase sous cutanée ou furonculoïde est une ectoparasitose causée par le cheminement cutané ou sous cutané de larves de divers diptères [1]. C'est une affection endémique en zone tropicale et l'agent causal est le plus souvent Cordylobia anthropophaga ou ver de Cayor [2]. Laspect clinique est une lésion furonculoïde [3]. Elle peut survenir à tout âge. A notre connaissance, très peu de cas de myiases sous-cutanés chez le nourrisson ont été rapportés dans la littérature médicale [4]. Nous en rapportons une observation chez un nourrisson de deux mois remarquable par sa présentation clinique simulant des furoncles multiples.

\section{OBSERVATION}

Il s'agissait d'un nourrisson âgé de deux mois, sans antécédents pathologiques particuliers, au statut vaccinal à jour. Il était reçu pour des lésions nodulaires multiples sensibles à la palpation, évoluant depuis 5 jours. Son état général était conservé, les constantes normales avec un bon développement psychomoteur. L'examen dermatologique retrouvait trois nodules inflammatoires centrés par un orifice blanchâtre ne laissant pas sourdre de liquide, localisés au niveau du thorax, le dos et la fesse (Fig. 1 - 3). Le reste de l'examen clinique était normal notamment sans adénopathies satellites. La compression bi-digitale d'un nodule au niveau du tronc avait permis l'extraction

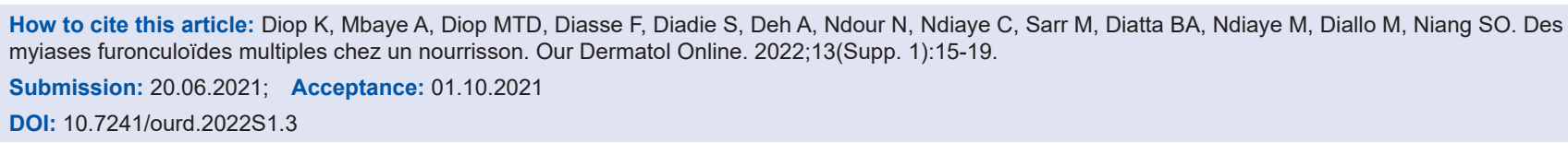


d'un asticot, laissant une ulcération de 5mm (Fig. 4). Le diagnostic de myiases furonculö̈des multiples était retenu devant la présentation clinique et la mise en évidence de larves à la compression bi-digitale. L'examen parasitologique de la larve n'a pas pu être réalisé. Le traitement consistait à l'extraction manuelle des larves et des soins locaux (Fig. 5). Lévolution était favorable au bout de deux semaines, marquée une cicatrisation complète des lésions.

\section{DISCUSSION}

Les myiases (du grec myia $=$ mouche $)$ sont des zoonoses résultant de l'infestation de l'homme ou d'animaux vertébrés par diverses espèces de larves de diptères [5]. La forme la plus commune est la myiase sous cutanée ou furonculoïde comme rapportée dans notre cas, qui est le plus souvent causée par Cordylobia anthropophaga en Afrique subsaharienne (ver de Cayor) et par Dermatobia hominis en Amérique latine (ver macaque) [2]. On distingue également la myiase cavitaire (ou luminale) ou des conduits naturels, la myiase des plaies, la myiase rampante et la myiase intestinale qui reste exceptionnelle [3]. Les myiases sous-cutanées sont rares et sousestimées, représentant $0,9 \%$ des ectoparasitoses [6]. Cette affection peut survenir à tout âge [7]. A notre connaissance, en Afrique Subsaharienne, très peu de cas de myiases sous-cutanées chez le nourrisson ont été rapportés dans la littérature médicale [4]. Cette rareté pourrait s'expliquer par une moindre exposition aux facteurs de risque chez le nourrisson. Les larves infestent le plus souvent les animaux domestiques. L'homme est un hôte accidentel.

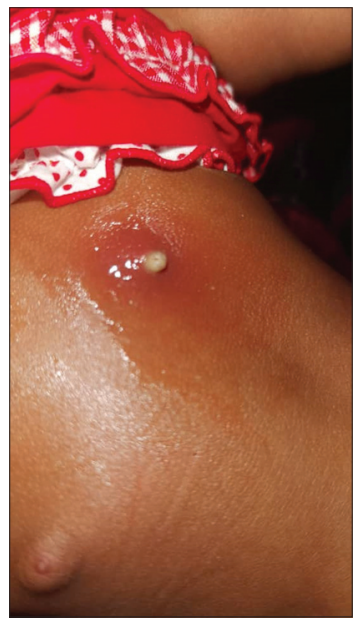

Figure 1: Inflammatory nodule centered by a whitish opening in the thorax.
L'infestation par les larves de C. anthropophaga survient après contact avec des oufs déposés sur du sable humide ou sur du linge ayant séché à l'air libre et non repassé. Après trois jours de maturation, les oufs libèrent une larve de stade 1 se déplaçant activement à la recherche d'un hôte. Au contact de la peau, la larve pénètre activement l'épiderme et se développe pendant une dizaine de jours dans le tissu sous cutané, en passant par trois stades larvaires. Les crochets buccaux de la larve permettent sa fixation chez l'hôte et son alimentation. Après des semaines de maturation, elle quitte la peau et atteint le sol pour se transformer en mouche [8]. Dans notre cas, le mode de contamination serait indirect par l'intermédiaire du linge ayant séché à l'air libre et non repassé, expliquant la topographie des lésions au niveau des zones couvertes.

Le diagnostic de la myiase sous-cutané est essentiellement clinique. Laspect clinique est une papule ou un nodule

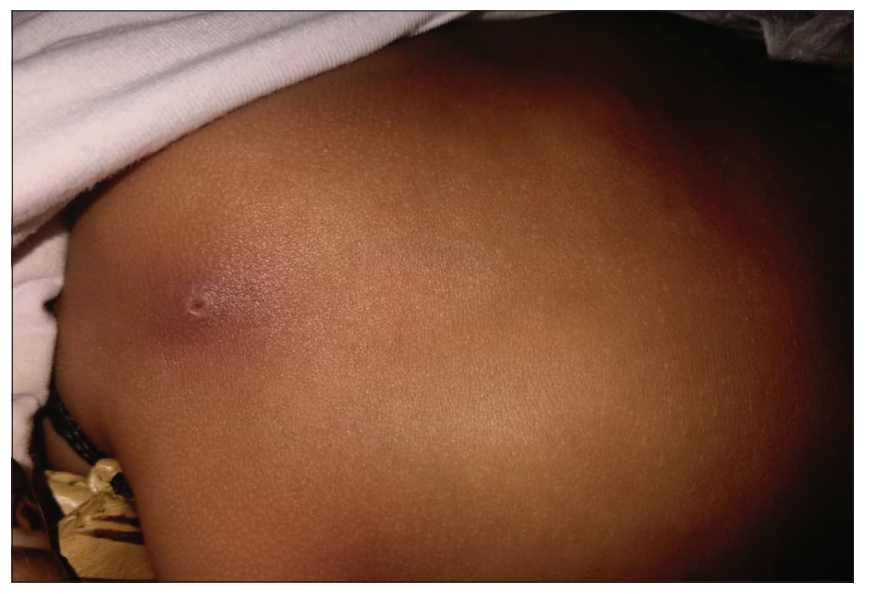

Figure 2: Inflammatory nodule on the back.

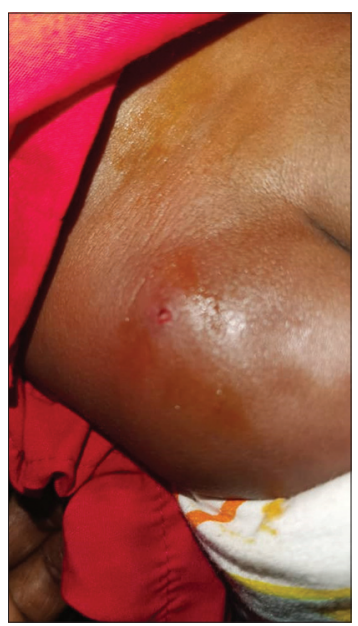

Figure 3: Inflammatory nodule of the buttock. 


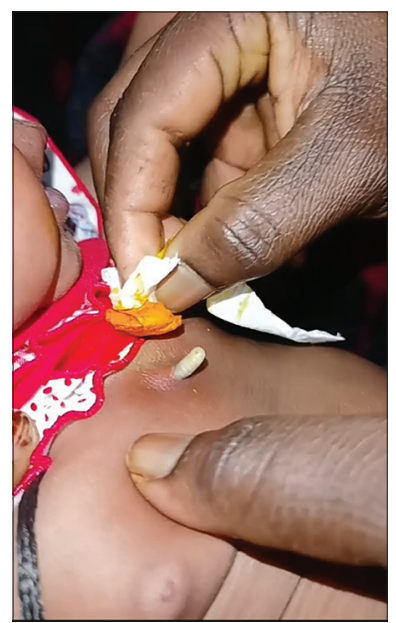

Figure 4: Manual extraction of Cordylobia Anthrophoga larva.

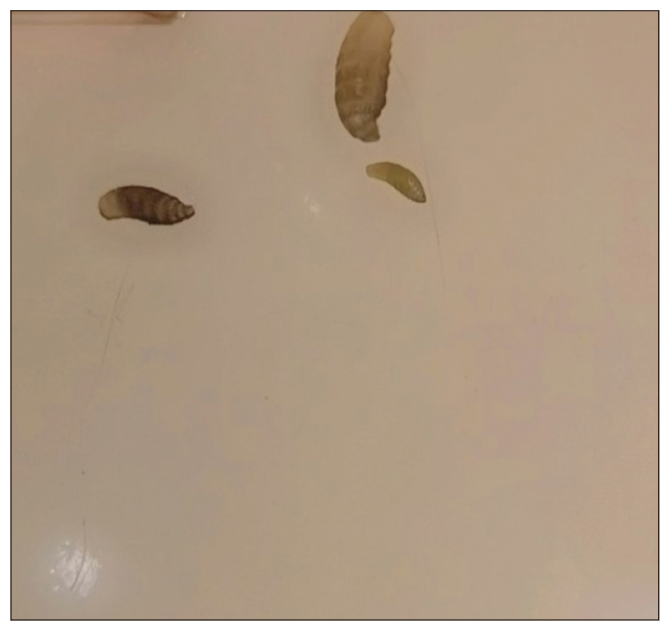

Figure 5:Larvae of Cordylobia Anthrophoga.

érythémateux parfois inflammatoire centré par un orifice blanchâtre comme observé dans notre cas [1,2]. Lexamen parasitologique de la larve permet de mettre en évidence l'espèce responsable qui est le plus souvent Cordylobia anthropophaga ou ver de Cayor en zone tropicale. La présentation clinique notait dans notre cas à type de lésions furonculoïdes multiples est inhabituelle et prêtait à confusion avec des furoncles ou des abcès multiples. Mais, le contexte épidémiologique et surtout l'examen minutieux des lésions à type de papules érythémateux centré par un orifice blanchâtre nous ont permis de retenir le diagnostic de myiases sous cutanées multiples.

Le traitement de la myiase sous-cutané dépend du stade larvaire $[9,10]$. Au stade initial de l'infection, la prise en charge consiste à asphyxier la larve par une substance occlusive (vaseline, vernis à ongles, huile de paraffine, huile de pétrole, lard, graisse de porc ou autres corps gras, pâte à mâcher.) $[2,9,10]$. Les corps occlusifs ont pour but de créer une asphyxie de la larve et forcer cette dernière à se diriger vers la surface de la peau pour trouver de l'air [10]. Il devient alors plus facile de l'extraire en appliquant une pression latérale autour de la lésion Lextraction manuelle à l'aide d'une pince ou par compression bidigitale est indiquée lorsque la maturation larvaire est complète comme réalisée dans notre cas. Enfin, l'extraction chirurgicale demeure une exception. Elle est préconisée en cas d'impossibilité de retirer la larve manuellement $[1,2,10]$. Elle doit être pratiquée de manière stérile et la larve doit être retirée entière. En cas de rupture de la larve, le patient risque de développer une réaction d'hypersensibilité et, plus tard, une réaction locale à corps étranger. Un traitement médicamenteux antiparasitaire n'est pas nécessaire, bien que l'ivermectine semble efficace. La complication principale de la myiase furonculoïde est la surinfection bactérienne [10]. La prophylaxie consiste à laver le linge de manière rigoureuse, à le mettre à sécher en plein soleil, et à le repasser avec un fer très chaud pour détruire les œufs $[1,2]$.

\section{CONCLUSION}

Nous rapportons une observation de myiase sous cutanée particulière par la survenue chez un nourrisson de deux mois et sa présentation clinique trompeuse à type de lésions furonculoïdes multiples. La prévention consiste à repasser le linge avant utilisation.

\section{Consent}

The examination of the patient was conducted according to the principles of the Declaration of Helsinki.

The authors certify that they have obtained all appropriate patient consent forms, in which the patients gave their consent for images and other clinical information to be included in the journal. The patients understand that their names and initials will not be published and due effort will be made to conceal their identity, but that anonymity cannot be guaranteed.

\section{REFERENCES}

1. Mbaye PA, Sagna A, Ndong A, Ndoye NA, Ndour O, Ngom G. [Scrotal myiasis at the child: Case study]. Afr J Urol. 2018;24:399_ 401.

2. Sarr A, Sow D, Diao B, Ndoye AK, Gueye O, Ba O, et al. [Preputial myiasis: about one case]. Andrologie. 2011;21:260-2.

3. Faida A, Rim A, Najeh B, Ali M, Ghazi K, Bassem L, et al. Des furonroncles résistants aux anti- biotiques: penser à la myiase ! Pan African Med J. 2013;15:41. 


\section{www.odermatol.com}

4. Deheck E, Nzungu PN, Cailliez JC, Guevart E, Delhaes L, DeiCas E, et al. Cordylobia anthropophaga (Diptera: Calliphoridae) Outside Africa: A case of furuncular myiasis in a child returning from Congo. J Med Entomol. 2005;42:187.

5. Hochedez P, Caumes E. [Common skin diseases in returning travelers], Lett Infectiol. 2008;XXIII:87-121.

6. Diabaté A, Kourouma HS, Kouabenan AAS, Gué I, Vagamon B, Aka BR. Profil l épidémiologique, clinique et évolutif des infections parasitaires cutanées superfi cielles en milieu hospitalier en Côte d'Ivoire, Rev Int Sc Med-RISM. 2018;20:1.

7. Ikrame B, Najwa B, Hanaa R, Sofi a N, Razika B, Benbouzid MA, et al. Myiase auriculaire humaine: à propos de deux cas. PAMJ Clin Med. 2021;51-2.

8. Devienne P, Bobard P, Pinhas C. Le ver de Cayor, agent d'une myiase furonculeuse. Insectes. 2004;35:23-4.

9. Elsendoorn A, Landron C, Goudet V, Pénin G, Roblot F. [An erysipelas revealing infection by Dermatobia hominis]. Med Mal Infect. 2010;40:45-7.

10. Hirsch G, Jeandel R, Biechler M, Boivin JF, Hillion B. Myiase furonculoïde à Dermatobia hominis: diagnostic fortuit sur la coupe macroscopique extemporanée d'un nodule cutané, Ann Dermatol Venereol. 2015;142:776-9.

Copyright by Khadim Diop, et al. This is an open-access article distributed under the terms of the Creative Commons Attribution License, which permits unrestricted use, distribution, and reproduction in any medium, provided the original author and source are credited.

Source of Support: Nil, Conflict of Interest: None declared. 